\title{
Wave processes in determining mechanical characteristics of soils
}

\author{
Karim Sultanov ${ }^{1 *}$, Pavel Loginov ${ }^{2}$, Sabida Ismoilova $^{2}$ and Zulfiya Salikhova ${ }^{2}$ \\ ${ }^{1}$ Tashkent Institute Irrigation and Agricultural Mechanization Engineers \\ ${ }^{2}$ Institute of Mechanics and Seismic Stability of Structures named after M.T. Urazbayev of the \\ Academy of Sciences of the Republic of Uzbekistan
}

\begin{abstract}
Mechanical characteristics of soils under dynamic and static loads are determined in laboratory conditions on special devices. Dynamic loads in the devices are initiated by an impact on a soil sample. Under the impact the waves are initiated in soil; they significantly affect the stressstrain state of soil samples placed in the device. Depending on the parameters of the impact load in the device, in different sections of soil sample there arise the stress-strain states, different in quality and quantity. Mechanical characteristics of soil, determined by this stress-strain state, also differ. The effect of stress-strain state of soil on its mechanical characteristics can be estimated theoretically. The initiation of the wave process and dynamic stress-strain state in soil sample placed in the device can be theoretically examined in detail. In this regard, the wave problem is set, which corresponds to the statement of experiments on the device of dynamic loading of soil. The law of soil strain is taken as an elasticviscoplastic one. Numerical solution of wave equations is obtained by the finite difference method. Based on the analysis of stress-strain state of soil in various sections, obtained by numerical calculations, the condition is derived under which the effect of wave processes on mechanical characteristics of soils is eliminated. This condition (formula) establishes the relationship between the wavelength, the velocity of wave propagation in soil, the thickness of the soil sample in the device and the duration of dynamic load.
\end{abstract}

\section{Introduction}

The reliability of buildings and structures is directly related to the strength of soil on/in which they are located. The soil strength depends on mechanical characteristics of soil. So, a reliable determination of mechanical characteristics of soils is an important problem of the reliability and seismic resistance of buildings and structures. Mechanical characteristics of soil are related to the laws of soil strain. These characteristics include the modulus of elasticity, Poisson's ratio, unloading modulus, soil viscosity coefficient, etc. Mechanical characteristics of soil are mainly determined by experiments. Mechanical characteristics of soils based on specific elastic-viscoplastic models of soils are defined in [1 - 5] under static and dynamic loads in laboratory and field conditions.

\footnotetext{
*Corresponding author: sultanov.karim@mail.ru
} 
The complex laws of strain in soils and rocks [6-10], applied problems of destruction [11], a comparative analysis of mechanical characteristics determined by different methods [12], nonlinear models of soil strain [13 - 18] and non-one-dimensional problems of seismic stability of earth structures - all these aspects require experimental data on mechanical characteristics of soils and rocks, especially when they are subjected to dynamic loads.

The values of mechanical characteristics of soils in calculating the seismic stability of underground structures [5] are of particular relevance. Without the knowledge of necessary mechanical characteristics of soils under dynamic strain, it is impossible to estimate the quantitative parameters of the waves propagating in soil media and rocks $[1-5,16]$. The reliability of the values of mechanical characteristics of soils is also very important when solving the problems of seismic resistance of above-ground and underground structures [19, 20].

In field experiments it is almost impossible to get the repetitions of exactly the same (in quantity and quality) experiments, which make possible the statistical processing of results. The latter aspect is very important to prove the reliability of experimental results.

The authors in [3] have created a special device for dynamic loading (DDL) to determine dynamic characteristics of soils. A soil sample of undisturbed and disturbed structure is tested for shock loads created by the falling of a weight. The DDL works on the principle of a compression device to test static loads in soils. The design description of the DDL and the principle of its operation are given in detail in [3]. The method of experimental determination of mechanical characteristics of soils proposed in [3] allows serial experiments, the results of which are then processed using the mathematical statistics methods. As a result, essentially reliable experimental data are obtained for determining mechanical characteristics of soils under dynamic loads [3].

However, another problem arises when conducting experiments on dynamic compression of soil samples on the DDL. It lies in the fact that the stress measurement results are influenced by the wave processes that occur in a soil sample when it is subjected to a shock load. To accurately measure the stresses under dynamic uniaxial compression of soil on the DDL, it is necessary to ensure the quasistatic nature of the process of soil strain. Quasistatic nature of the process of dynamic strain is satisfied under conditions $\frac{\mathrm{d} \sigma}{\mathrm{d} x}=0$ and $\frac{\mathrm{d} \varepsilon}{\mathrm{d} x}=0$; where $\sigma-$ is the longitudinal stress on $x$-axis of compression, $\varepsilon-$ is the longitudinal strain on $x$-axis.

The aim of this work is a theoretical study of the wave processes in a soil sample placed in the DDL soil receiving chamber and determination of the condition for the quasistatic nature of the process of dynamic strain in a soil sample based on the analysis of wave parameters in soil.

\section{Methods}

The devices UDN-100 and UDN-150 described in [3] have a cylindrical soil receiving chamber of a diameter of 0.1 or $0.15 \mathrm{~m}$ and a height of $0.03 \mathrm{~m}$, into which a cylindrical soil sample of undisturbed or disturbed structure is placed. The bottom plane of the chamber on which the soil sample rests is fixed. On the upper plane of the soil sample there is a piston, through which the dynamic load created by the impact of a weight free falling along the rod guides, is transferred [3].

The wave pattern corresponding to the statement of experiment on the DDL is quite complicated. On the $\mathrm{x}$-axis of a cylindrical soil sample, a load acting from the upper piston varies from zero to a maximum and then again to zero. According to the results obtained in 
[3], one of the conditions for ensuring the quasistatic behavior of the strain process is the ratio of the rise time of the load to the maximum and the fall time to zero. The rise time of the stress should always be less than the fall time. The change in stresses is presented in the form of a half-period of a sinusoid, in this case the rise time equals to the fall time. This is more rigid condition for ensuring the quasistatic nature of the process of soil strain on the DDL.

The load acts uniformly on the upper plane of the soil layer, located in the DDL soil receiving chamber, from which follows the one-dimensionality of dynamic and static processes of soil compression. The side walls of the soil receiving chamber have sufficient roughness that allows one to take the friction force between the soil and the side wall as equal to zero. Based on this, the soil friction forces on the side surface are neglected.

A layer of soil $0.03 \mathrm{~m}$ thick is located at a distance $0-x_{*}$ along the $x$-axis. At a distance $x=x_{*}$ there is an upper plane of the lower, absolutely rigid, fixed piston. This plane can also be considered as a fixed obstacle.

According to the results of experiments given above, the soil is considered to be an elastic-viscoplastic medium. At $t=0$, load $\sigma=\sigma(t)$ begins to act on the soil layer; this load obeys a sinusoidal law. A wave propagates through undisturbed soil. The front of this wave reaching the lower plane is reflected from it. Under load $\sigma=\sigma(t)$ plastic strains are formed in soil. Consequently, a plastic wave propagates in soil. After load $\sigma=\sigma(t)$ reaches its maximum, another front is formed - of maximum stresses in soil. At $\sigma=\sigma(t)=0$ the front of unloading wave propagates in soil. The fronts reflected from the lower piston $\left(x=x_{*}\right)$ and from the upper piston located at $x=0$, form a complex wave pattern.

Due to the fact that the load acting on the soil layer is continuous, these fronts are the lines of weak discontinuity, that is, on these fronts the wave parameters do not have jumps (discontinuities). Only the first derivatives of the wave parameters can have discontinuities on these fronts; therefore, they are called the weak discontinuity lines. This circumstance, as will be shown later, greatly simplifies the solution of theoretical problem corresponding to the dynamic compression of the soil layer on the DDL.

The linearity of wave fronts mainly depends on the specific type of the equation of state of soil. In the case of linearity or even piecewise linearity of the equation of state of soil, which determines mechanical characteristics of soil, the wave fronts remain linear. Otherwise, these fronts are nonlinear ones. In the case of nonlinearity of the fronts, the problem of dynamic compression of a soil sample on the DDL becomes significantly complicated. Therefore, the wave pattern and the statement of the problem corresponding to the case under consideration directly depend on the equation of state or the law of soil strain, on the basis of which mechanical characteristics of soil are determined.

In $[3,4,15]$ it is shown that even at loads of $0.3-0.5 \mathrm{MPa}$, the process of dynamic strain of loess soil in experiments displays the elastic-viscoplastic properties of soils. Based on this, a soil model adequate to this process is chosen.

According to the analysis of the equation of state of soil $[1-5,15]$, the most perfect law of soil strain, taking into account the plastic strain of soil and its viscous properties at volume changes, is the law proposed in [4]. Consider this law in relation to the process of strain of loess soils on the DDL.

The model of elastic-viscoplastic medium [4] has the following form:

$$
\frac{\mathrm{d} \varepsilon}{\mathrm{d} t}+\mu \varepsilon=\frac{\mathrm{d} \sigma}{E_{D} \mathrm{~d} t}+\mu \frac{\sigma}{E_{S}} \text { at } \frac{\mathrm{d} \sigma}{\mathrm{d} t}>0, \frac{\mathrm{d} \varepsilon}{\mathrm{d} t}>0
$$




$$
\begin{gathered}
\frac{\mathrm{d} \varepsilon}{\mathrm{d} t}+\mu \varepsilon=\frac{\mathrm{d} \sigma}{E_{R} \mathrm{~d} t}+\mu \sigma\left(\frac{1}{E_{S}}-\frac{1}{E_{D}}+\frac{1}{E_{R}}\right)+\mu \sigma_{m}\left(\frac{1}{E_{D}}-\frac{1}{E_{R}}\right) \text { at } \frac{\mathrm{d} \sigma}{\mathrm{d} t}<0, \frac{\mathrm{d} \varepsilon}{\mathrm{d} t}>0 \\
\frac{\mathrm{d} \varepsilon}{\mathrm{d} t}=\frac{\mathrm{d} \sigma}{E_{R} \mathrm{~d} t} \text { at } \frac{\mathrm{d} \sigma}{\mathrm{d} t}<0, \frac{\mathrm{d} \varepsilon}{\mathrm{d} t}<0
\end{gathered}
$$

where $E_{D}$ - is the modulus of dynamic compression of soil at $\mathrm{d} \varepsilon / \mathrm{d} t \rightarrow \infty, E_{S}-$ is the modulus of static compression of soil at $\mathrm{d} \varepsilon / \mathrm{d} t \rightarrow 0, E_{R}-$ is the unloading modulus, $\mu-$ is the viscosity parameter, related to the viscosity coefficient by the ratio

$$
\mu=\frac{E_{D} E_{S}}{\eta\left(E_{D}-E_{S}\right)}
$$

where $\eta$ - is the soil viscosity coefficient at volume changes, $\sigma_{m}-$ is the maximum stress in the soil particle.

The strain $\varepsilon$, as applied to the experiments on the DDL-150, uniquely determines the change in volume of the soil layer. Therefore, it can be taken as a volumetric strain, and $\sigma-$ as the pressure. In this case, $\sigma=-P$, where $P-$ is the pressure. It follows that the equation of state of soil (1)-(3) is the law of variation of the spherical part of the stress tensor, that is, the law of volume strain of soil. From (1)-(3) it is seen that in this case the main mechanical characteristics of soil are $E_{D}, E_{S}, E_{R}$ and $\mu$ or $\eta$.

Until now, the values of these mechanical characteristics of soils or others (based on other equations of state) have been determined directly from the results of experiments based on soil compression diagrams.

To consider the wave process in soil and the dynamic strain of soil on the DDL, it is necessary to solve the equation of motion of soil, which has the form:

$$
\rho_{0} \frac{\partial v}{\partial t}-\frac{\partial \sigma}{\partial x}=0, \frac{\partial v}{\partial x}-\frac{\partial \varepsilon}{\partial t}=0
$$

where $\rho_{0}-$ is the initial density of soil, $v-$ is the velocity of the soil particles under compression.

The equation of one-dimensional motion of soil in the DDL (5) is successively closed by the equations of state of soil (1)-(3). In the closed system of equations (1)-(5), the unknowns are $\sigma, \varepsilon$ and $v$, called the parameters of the waves in soil or the parameters of the stress-strain state of soil in the DDL.

To solve the system of differential equations (1) - (5), initial and boundary conditions are necessary. The initial conditions of the problem are zero, since before the impact of the load, the soil on the DDL is at rest, that is, it is considered undisturbed.

The boundary conditions of the problem corresponding to the statement of the experiment are the following: at $x=0$ the upper plane of the soil layer on the DDL is affected by the load $\sigma=\sigma(t)$ through the motion of the upper piston; at $x=x_{*}$ the lower piston is fixed, $v=0$, i.e. the velocity of the soil particles at this boundary is zero.

Mathematical formulation of the boundary conditions is as follows:

$$
\begin{gathered}
\sigma=\sigma(t) \text { at } x=0,0<t<t_{*} \\
\sigma=0 \text { at } x=0, t>t_{*} \\
v=0 \text { at } x=x_{*}
\end{gathered}
$$

where $t *$ is the time of load effect.

On the front of the incident wave, the following condition is satisfied:

$$
\langle\sigma\rangle=0,\langle\varepsilon\rangle=0,\langle v\rangle=0 \quad \text { at } \quad x=c t
$$


where $c-$ is the velocity of longitudinal wave propagation in soil, $\langle\sigma\rangle,\langle\varepsilon\rangle,\langle v\rangle-$ are the jumps in wave parameters.

In the equations of state (1) - (3), the front line $x=c t$ and the lines of all other fronts are straight lines. This follows from the linearity of the equations that make up the law of soil strain (1) - (3).

Thus, the process of dynamic strain in the soil sample placed on the DDL is described by the system of equations (1) - (5). Having solved the system of equations (1) - (5) with boundary conditions (6) - (9) and zero initial conditions, the dynamic stress-strain state of soil can be determined. The system of equations (1) - (5) is a hyperbolic one [3, 5]. At present, it is not possible to obtain an analytical solution of these equations. Therefore, the approximate methods are used. Following [3,5], the differential equations in partial derivatives are reduced by the method of characteristics to the ordinary differential equations. Hyperbolic system (1) - (5) has real characteristics and characteristic relations.

The application of the finite difference method to ordinary differential equations improves the accuracy of the solution as compared to partial differential equations $[3,5]$.

The derivation of the equations of characteristics and characteristic relations is given in more detail in [4]. In [15], the solution of the problem of explosive wave interaction with a rigid moving obstacle in soil is considered. The problem we are considering here differs from [15] by the boundary conditions, the nature of the load acting on soil and the aim of the task.

Thus, the solution of the system of equations (1) - (5) with boundary conditions (6) (9), is obtained by the method of characteristics with the subsequent application of the method of finite differences. On the basis of the developed algorithm for solving the problem, a program for solving the problem in the Turbo Pascal algorithmic language has been created. The developed program is implemented on a computer. The stability of the algorithm for solving the problem is verified by a numerical experiment. The results of numerical experiments have shown that the stability of the computational scheme and algorithm is fully ensured and controlled by the Courant condition.

\section{Results}

The regularities of plane wave propagation in soil as in elastic-viscoplastic media are theoretically investigated in $[4,5,16]$. The solution of the theoretical problem according to the above method in the case when soil is a linear viscoelastic medium (a standard linear body), has been carried out numerically in $[4,16]$ using the method of characteristics. In [4, 16], the problems of wave interaction with a moving non-deformable obstacle in a viscoplastic medium - soil - are considered. The interaction of a wave with a deformable obstacle in a viscoelastic medium has been studied in [5].

The interaction of a continuous compression wave with a rigidly fixed obstacle in an elastic-viscoplastic medium has not been studied yet. Consequently, the solution of the problem considered here is obtained for the first time. The considered problem of the interaction of a plane continuous compression wave with a rigidly fixed obstacle in an elastic-viscoplastic medium - soil - is being investigated here on the DDL to substantiate a reliable determination of mechanical characteristics of soils. Nevertheless, the developed algorithm and the program for solving the problem allow us to investigate on the basis of the results of numerical solutions the change in the wave parameters in soil and the laws of soil strain, not only in relation to the DDL.

The main parameters (initial data) of the problem to conduct the calculations are: - the soil characteristics $-\gamma=E_{D} / E_{S}, \beta=E_{D} / E_{R}, \rho_{0}, c, \mu$;

- the load characteristics $-\sigma_{\max }, t_{*}$; 
- the distance from the initial section of soil to the obstacle $-x_{*}$.

The options for computer calculations at different values of these parameters are given in Table 1. They are selected based on the parameters of seismic load in loess soils [5]. Based on the results of experiments [5], the value of the maximum load $\sigma_{\max }$ for all options is taken as equal to $0.5 \mathrm{MPa}$. The initial density of soil $\rho_{0}$ is $1500 \mathrm{~kg} / \mathrm{m}^{3}$, the velocity of longitudinal wave propagation is $100 \mathrm{~m} / \mathrm{sec}$. Values $\rho_{0}$ and $c$ for option 6 have been changed: $\rho_{0}=2000 \mathrm{~kg}^{3} / \mathrm{m}, c=1000 \mathrm{~m} / \mathrm{sec}$. Option 6 corresponds to elastic-plastic soil, where the density of soil and, respectively, the velocity of longitudinal wave propagation in soil are more significant than the ones in elastic-viscoplastic soil [5].

Table 1. Options of numerical calculations and the values of initial data

\begin{tabular}{|c|c|c|c|c|c|}
\hline No of the option & $\gamma$ & $\beta$ & $x_{*}, m$ & $t_{*}, s$ & $\mu, s^{-1}$ \\
\hline 1 & 2 & 0.5 & 2.8 & 0.1 & 100 \\
\hline 2 & 4 & 0.5 & 2.8 & 0.1 & 100 \\
\hline 3 & 2 & 0.5 & 0.28 & 0.1 & 100 \\
\hline 4 & 2 & 0.5 & 0.03 & 0.1 & 100 \\
\hline 5 & 4 & 0.5 & 0.03 & 0.1 & 100 \\
\hline 6 & 1.05 & 0.5 & 0.03 & 0.1 & 1000 \\
\hline 7 & 2 & 0.25 & 0.03 & 0.01 & 100 \\
\hline 8 & 2 & 0.5 & 0.03 & 0.01 & 100 \\
\hline 9 & 2 & 0.5 & 0.03 & 0.001 & 100 \\
\hline
\end{tabular}

When choosing the options listed in Table 1 , virtual values of $\gamma, \beta, x_{*}, t_{*}, \mu$ have been taken into account according to $[3-5,16]$.

The value of the dynamic compression modulus is determined by formula $E_{D}=c^{2} \rho_{0}$, the static compression modulus by $E_{S}=E_{D} / \gamma$, and the unloading modulus by $E_{R}=E_{D} / \beta$.

Numerical solution of the problem is obtained in a dimensionless form. Then, it was converted to a dimensional form. Consider the results of calculations obtained on a computer.

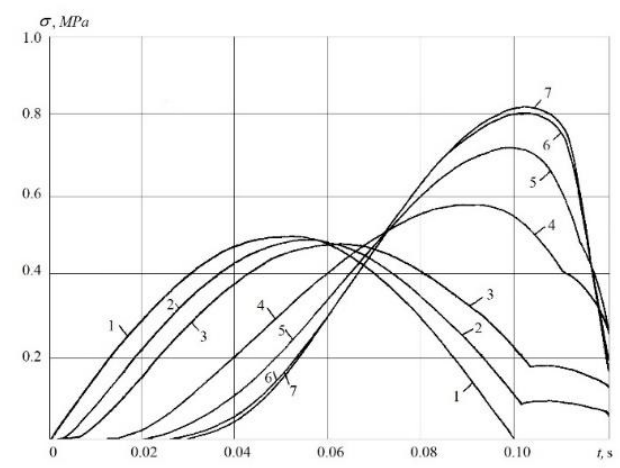

Fig. 1. Change in stresses in soil sections: 1) $x=0$;2) $x=0.28$; 3) $x=0.56$;

4) $x=1.15$; 5) $x=1.2$;6) $x=2.55$; 7) $x=2.8 \mathrm{~m}$ 


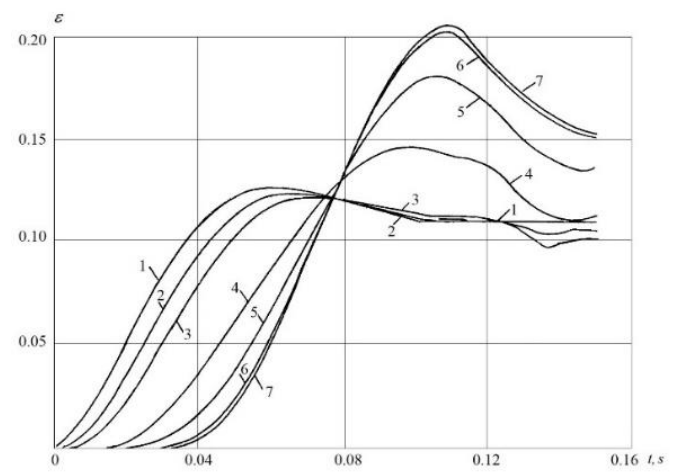

Fig. 2. Change in strain in soil sections: 1) $x=0$; 2) $x=0.28$; 3) $x=0.56$; 4) $x=1.15$;

$$
\text { 5) } x=1.2 \text {;6) } x=2.55 \text {;7) } x=2.8 \mathrm{~m}
$$

Figure 1 shows the change in the longitudinal stresses $\sigma$ over time $t$ for different sections of soil for option 2 (Table 1). Curves 1-7 refer to soil sections at $0 ; 0.28 ; 0.56$; $1.15 ; 1.2 ; 2.55$ and $2.8 \mathrm{~m}$, respectively. At $x=x_{*}=2.8 \mathrm{~m}$ there is a fixed and nondeformable obstacle (the lower piston of the DDL).

In theoretical calculations it is possible to locate the lower piston of the device at an arbitrary distance from the upper piston. In option 2, the obstacle (the lower piston) is specifically set aside at a distance of $x_{*}=2.8 \mathrm{~m}$ in order to study the patterns of change in the wave parameters in soil.

According to the conditions set, a half-period of sinusoidal load is acting on soil (Figure 1 , curve 1). At a distance of $x=2.83 \mathrm{~m}$ there is a lower piston of the device (an obstacle). In this option, at the load time $t_{*}=0.1 \mathrm{sec}$, the wave 3.5 times runs to the obstacle and back. As a result of the superposition of waves reflected from the lower and upper pistons, different stress values are observed on different sections of the soil layer. At the obstacle, the maximum stress is 1.6 times greater than in the initial section (curve 7). In other sections of soil, the maximum stress value is also greater than in the initial section. A similar pattern is observed in the dependencies $\varepsilon(t)$ (Figure 2). Here also, the maximum strain is achieved on the obstacle (curve 7). The values of residual strains in soil sections are essential. Curves 1 - 7 refer to the same distances as the ones in Figure1.

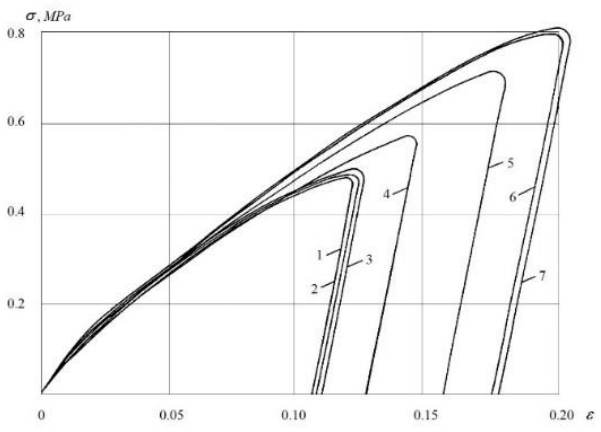

Fig. 3. Change in strain in soil sections: 1) $x=0$; 2) $x=0.28$; 3) $x=0.56$;

4) $x=1.15$; 5) $x=1.2$; 6) $x=2.55$;

7) $x=2.8 \mathrm{~m}$

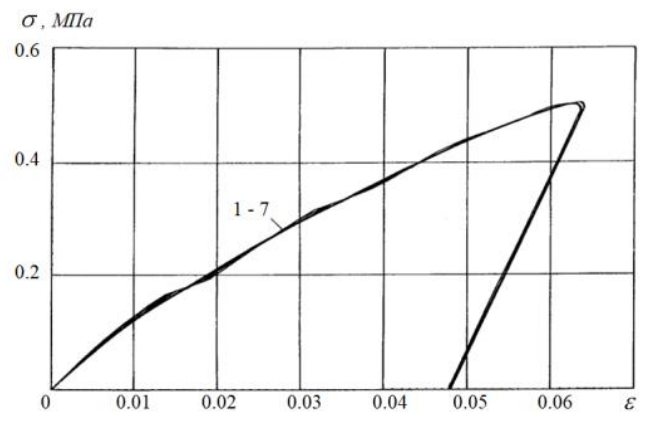

Fig. 4. Stress-strain dependence for soil sections:

1) $x=0$; 2) $x=0.28$; 3) $x=0.56$;

4) $x=1.15$; 5) $x=1.2$;6) $x=2.55$;

7) $x=2.8 \mathrm{~m}$ 
Dependences of $\sigma(t)$ and $\varepsilon(t)$ in Figures 1 and 2 show that the changes in stresses and strains are different under dynamic loads in different sections of soil. This circumstance must be taken into account when conducting similar experiments.

From Figures 1 and 2 it is seen that the wave processes in soil in this option present a complex pattern. When conducting field experiments, as in $[5,16]$, at a considerable thickness of a soil layer, to determine mechanical characteristics of soil under dynamic load, it is necessary to take into account the possibility of formation of complex wave processes in soil.

Compression diagrams for the considered soil sections (option 2) are shown in Figure 3. Here, an elastic-viscoplastic strain in soil is observed in all sections of soil. However, quantitatively dependences $\sigma(\varepsilon)$ are different for different sections of soil. At the same values of initial data, on which the calculations of option 2 have been carried out, significant residual strains in soil are observed. The maximum stress values are slightly behind the maximum strain values. Mechanical characteristics of soils, determined on the basis of curves $1-7$, are different.

In general, as shown by the results of the above numerical solutions, the patterns of changes in the wave parameters in soil depend on the characteristics of soil, on the thickness of the soil layer and, of course, on the characteristics of dynamic load. The latter is especially important to ensure the quasistatic nature of the process of soil strain in experiments. This issue is studied on calculation examples of options 4 - 9 (Table 1).

\section{Discussion}

As shown above, the quasistatic nature of the process of dynamic strain in soil in the DDL depends on the thickness of the soil layer. At essential thickness of the layer $x_{*}>0.3 \mathrm{~m}$ and the time of load effect $t * \leq 0.1 \mathrm{sec}$, we can assume that the quasistatic nature of the process of soil strain is not provided (options 1 and 2). This means that the values of stresses and strains in different sections of soil will differ significantly. To refer these differences in experiments to the scattering of experimental data will be incorrect and will lead to incorrect results in statistical processing. Therefore, it is very important to exclude the differences in dependences of $\sigma(t)$ and $\varepsilon(t)$ associated with the influence of the waves reflected from the lower piston. In experiments, a complete coincidence of dependences of $\sigma(t)$ and $\varepsilon(t)$, fixed by sensors installed above and below the soil layer, is necessary.

This is ensured, first of all, by the condition of the quasistatic nature of the process of dynamic strain in soil. In other words, when a dynamic load is applied, the soil layer strains almost statically, that is, at all its points the stress and strain values will be the same. Such dynamic strain of soil is called a quasistatic strain.

The thickness of the soil layer in experiments on the DDL is $x_{*}=0.03 \mathrm{~m}$. So, when choosing options $4-9$ (Table 1 ), $x_{*}$ is assumed to be $0.03 \mathrm{~m}$. Using the calculation example of option 4 , consider the change in the wave parameters in the soil layer of $3 \mathrm{~cm}$ thick and the load time $t *=0.1 \mathrm{sec}$. In options $4-9$, changes in the wave parameters are considered in the following sections of the soil layer $x=0 ; 0.0028 ; 0.0056 ; 0.015 ; 0.02$; 0.0255 and $0.03 \mathrm{~m}$. In Figure 4 these distances refer to curves $1-7$, respectively. 
Figure 4 shows the dependencies of $\sigma(\varepsilon)$ for the above sections of the soil layer (option 4, Table 1). From Figure 4 it can be seen that with a load time of $t_{*}=0.1 \mathrm{sec}$, the dependences of $\sigma(\varepsilon)$ for all sections of soil are absolutely the same. This proves the complete quasistatic nature of the process of soil strain on the DDL under dynamic load.

Calculation results of the options given in Table1, allow us to estimate the quasistatic nature of the process of soil strain on the DDL. To do this, the half the wavelength, propagating in soil, is determined according to the following formula:

$$
\lambda=c t
$$

Introduce the ratio of the half the wavelength $\lambda$ to the thickness of the soil layer $\delta$. The data obtained for options 1 - 9 are shown in Table 2 .

Table 2.

\begin{tabular}{|c|c|c|c|c|c|}
\hline No of the option & $\boldsymbol{c}, \mathbf{m} / \boldsymbol{s}$ & $\boldsymbol{t}_{*}$ & $\boldsymbol{x}_{*}, \mathbf{m}$ & $\boldsymbol{\lambda} \mathbf{, \mathbf { m }}$ & $\boldsymbol{\lambda} / \boldsymbol{x}_{*}$ \\
\hline 1 & 100 & 0.1 & 2.8 & 10 & 3.5 \\
\hline 2 & 100 & 0.1 & 2.8 & 10 & 3.5 \\
\hline 3 & 100 & 0.1 & 0.28 & 10 & 35 \\
\hline 4 & 100 & 0.1 & 0.03 & 10 & 333 \\
\hline 5 & 100 & 0.1 & 0.03 & 10 & 333 \\
\hline 6 & 1000 & 0.1 & 0.03 & 100 & 3333 \\
\hline 7 & 1000 & 0.1 & 0.03 & 10 & 333 \\
\hline 8 & 100 & 0.01 & 0.03 & 1 & 33 \\
\hline 9 & 100 & 0.001 & 0.03 & 0.1 & 3.3 \\
\hline
\end{tabular}

According to the results of calculations (of options 1 - 9), for options 1, 2, 9 the quasistatic nature of the process of soil strain on the DDL is not clearly observed. For options 3 and 8 the quasistatic process is observed satisfactorily. For options $4-7$ the quasistatic process is observed with high accuracy.

Based on this and the data of Table 2, the following condition must be met to maintain the quasistatic nature:

$$
\lambda / x_{*}=\lambda / \delta_{o}>50
$$

At the ratio of the half the wavelength to the thickness of the soil layer $\delta$ more than 50 , the quasistatic nature of the strain process under dynamic compression of soil in the DDL is fully ensured. The higher the ratio of $\lambda / \delta$, the higher the quasistatic nature of the process of soil strain. Consequently, the reliability of experimental results obtained on the DDL and mechanical characteristics of soil determined on the basis of these experiments increases.

\section{Conclusion}

Numerical solution of the problem of dynamic compression of a soil layer, as an elasticviscoplastic medium, has been obtained as applied to the experiment statement on the DDL.

The wave processes in soil are studied at various values of the problem parameters, which characterize soil properties, dynamic loads and the experimental setup of the DDL. The calculation results have established the degree of the effect of the problem parameters on the values of stress, strain, particle velocity and soil displacement.

It has been established that a change in physical and mechanical properties of soil has practically no effect on the quasistatic nature of the process of soil strain in experiments on the DDL. 
Based on the analysis of the results of numerical solutions, the conditions (formula) are derived to ensure the quasistatic nature of the process of dynamic strain of soil in experiments on the DDL.

\section{References}

1. A.A. Vovk, B.V. Zamyshlyaev, L.S. Yevterev, I.V. Belinsky, A.V. Mikhalyuk, Behavior of soils under the influence of impulse loads (Kiev: Naukova Dumka, 1984)

2. B.V. Zamyshlyaev, L.S. Yevterev, Models of dynamic strain and damage of soil medium (Moscow: Nauka, 1990)

3. G.V. Rykov, A.M. Skobeev, Stress measurement in soils under short-term loads (Moscow: Nauka, 1978)

4. G.M. Lyakhov, Fundamentals of the dynamics of blast waves in soils and rocks (Moscow: Nedra, 1974)

5. K.S. Sultanov, Wave theory of seismic resistance of underground structures (Tashkent: Fan, 2016)

6. E.K. Mojtaba, T. Hossein, K. Nasser, International Journal of Geomechanics, 14, 2 (2014)

7. G. Buscarnera, G. Dattola, Soils and Foundations, 56, 1 (2016)

8. J. Monnet, European Journal of Environmental and Civil Engineering, 16, 6 (2012)

9. J. Monnet, European Journal of Environmental and Civil Engineering, 16, 6 (2012)

10. A.G. Shashkin, Soil Mechanics and Foundation Engineering, 48, 4 (2011)

11. I.T. Mirsayapov, I.V. Koroleva, Soil Mechanics and Foundation Engineering, 53, 1 (2016)

12. A.N. Levandovskiy, B.E. Melnikov, A.A. Shamkin, Magazine of Civil Engineering, 1, 69 (2017)

13. Ju.A. Kalugina, D. Keck, Ya.A. Pronozin, Magazine of civil Engineering, 7, 75 (2017)

14. K.S. Sultanov, Journal Applied Mathematics and Mechanics, 62, 3 (1998)

15. K.S. Sultanov, B.E. Khusanov, Soil Mechanics and Foundation Engineering, 38, 3 (2001)

16. G.M. Lyakhov, Waves in soils and porous multi-component media (Moscow: Nauka, 1982)

17. K.S. Sultanov, B.E. Khusanov, Soil Mechanics and Foundation Engineering, 39, 3 (2002)

18. K.S. Sultanov, Journal Applied Mathematics and Mechanics, 66, 1 (2002)

19. M.M. Mirsaidov, T.Z. Sultanov, Soil Mechanics and Foundation Engineering, 49, 6 (2013)

20. T.Z. Sultanov, D.A. Khodzhaev, M.M. Mirsaidov, Magazine of Civil Engineering, 45, 1 (2014) 